\title{
Computed tomography quantification of airway remodelling in normal ageing subjects: a cross-sectional study
}

To the Editor:

Airway remodelling inducing structural and functional decline is a main factor leading to clinical disablement in chronic obstructive pulmonary disease (COPD) [1]. However, whether airway remodelling is also a potential feature of the normal healing process in ordinary lifelong environmental exposure and chronic endogen damage due to senescence is not well elucidated [2]. The relationship between ageing and development of COPD is complex. On one hand, while there is a higher prevalence of COPD in the elderly, ageing is not the direct cause of COPD; on the other hand, it does increase the susceptibility of the lung to extrinsic damage [3].

To avoid misinterpretation in chest computed tomography (CT) analysis, physiological, age-related changes [4] should be recognised. Thus, the purpose of this prospective study was to evaluate whether airway remodelling resulting from the normal ageing process can be assessed in vivo using software-assisted CT quantification, with correlation to pulmonary function testing (PFT).

This study was conducted with the approval of the local research ethics committee. Participants were required to be asymptomatic, aged from 60 to 90 years, and nonsmokers for $\geqslant 20$ years, with a cumulative history of $<10$ pack-years of tobacco use.

Subjects were excluded if they met any of the following criteria: cognitive disorders; past or current respiratory disease; congestive heart failure or myocardial infarction during the past year; chest radiotherapy; current or previous treatment with medication known to cause lung toxicity; former occupational exposure to asbestos or coal dust, or having any other occupational activity known to be related to lung disease.

CT acquisitions were spirometrically controlled (Winspiro, Waukesha, WI, USA) and performed on a 64 detector row CT scan unit (LightSpeed VCT; GE Healthcare, Little Chalfont, UK) without contrast material. The whole chest volume was acquired at total lung capacity for inspiratory CT, whereas for expiratory CT, only four incremental slices were performed at residual volume: one through the upper lobes, one through the tracheal carina, and two between the tracheal carina and the right diaphragmatic dome.

Data were anonymously processed by two radiologists (S. Bommart and F. Klein). The inspiratory CT acquisition was computed using commercially available CT software (Thoracic VCAR; GE Healthcare). This software performs automated tracking of the airways providing cross-sectional views perpendicular to each bronchus long axis, and subsequently measures the bronchial lumen and wall. Proximal airway analysis included three different segmental bronchi: the right apical bronchus (B1), the anterior basal bronchus (B8) and the posterior basal bronchus (B10) were arbitrarily chosen to be the site of the automated measurements. The lumen area (LA), wall area (WA), wall thickness (WT) and total diameter of the bronchus (WD) were automatically displayed at a site chosen by the radiologist, at mid-distance between the origin of the segmental bronchus and its first division. WT/WD and WT/WA ratios for each segment, and the square root of WA for a standardised airway with an internal perimeter of $10 \mathrm{~mm}$ (Pi10) were calculated [5]. Air trapping was quantified using software (Myrian; Intrasense, Montpellier, France) based on threshold techniques. Expiratory/inspiratory ratios of mean lung density (MLD) were calculated [6].

Subjects also underwent spirometry using a whole-body plethysmograph (Body Box; Medisoft, Dinant, Belgium) according to current guidelines [7]. Single-breath nitrogen testing (SBNT) was performed using the Vmax apparatus (Sensormedics, Yorba Linda, CA, USA) [8, 9].

The airway measures were modelled as a function of age. Univariate analyses were carried out using Spearman correlations and linear regressions.

This single-centre observational study enrolled 101 consecutive community-based volunteers, recruited between August 2009 and April 2012. 11 were unable to perform adequate respiratory manoeuvres during 
TABLE 1 Univariate relationship of each of the airway ratios and pulmonary function tests with age

\begin{tabular}{|c|c|c|c|c|c|}
\hline \multirow[t]{2}{*}{ Age-related tested variable } & \multirow[t]{2}{*}{ Correlation (CI) } & \multicolumn{4}{|c|}{ Regression } \\
\hline & & $\begin{array}{l}\text { Regression } \\
\text { coefficient }\end{array}$ & SE & p-value & $\mathbf{R}^{\mathbf{2}}$ \\
\hline $\mathrm{FEV}_{1}$ & $-0.39(-0.57--0.19)$ & -4.92 & 1.11 & $<0.001$ & 0.18 \\
\hline FVC & $0.03(-0.19-0.25)$ & -0.07 & 0.27 & 0.794 & $<0.01$ \\
\hline $\mathrm{FEV}_{1} / \mathrm{FVC}$ & $-0.26(-0.46-0.04)$ & -0.19 & 0.13 & 0.151 & 0.02 \\
\hline $\mathrm{MEF}_{50 \%}$ & $-0.32(-0.51--0.10)$ & -1.83 & 0.62 & 0.004 & 0.08 \\
\hline $\mathrm{MEF}_{25-75 \%}$ & $0.05(-0.18-0.27)$ & 0.07 & 0.67 & 0.911 & $<0.01$ \\
\hline RV & $0.14(-0.08-0.35)$ & 1.90 & 1.43 & 0.186 & 0.02 \\
\hline FRC & $-0.07(-0.27-0.16)$ & $-0,72$ & 1,17 & 0.541 & $<0.01$ \\
\hline TLC & $-0.20(-0.40--0.16)$ & -1.69 & 0.69 & 0.016 & 0.06 \\
\hline CV & $-0.37(-0.54--0.16)$ & -3.17 & 0.95 & 0.001 & 0.11 \\
\hline $\mathrm{dN}_{2}$ & $0.31(0.10-0.50)$ & 1.05 & 0.35 & 0.003 & 0.09 \\
\hline$E / I_{M L D}$ & $0.48(0.29-0.64)$ & 119.01 & 24.83 & $<0.001$ & 0.20 \\
\hline WT/WD B1 4th generation & $0.08(-0.14-0.30)$ & 35.52 & 37.34 & 0.344 & $<0.01$ \\
\hline WT/WA B1 4th generation & $0.10(-0.12-0.32)$ & 41.30 & 27.66 & 0.139 & 0.02 \\
\hline WT/WD B1 5th generation & $0.17(-0.05-0.38)$ & 70.93 & 37.60 & 0.062 & 0.04 \\
\hline WT/WA B1 5th generation & $0.10(-0.13-0.31)$ & 24.75 & 15.76 & 0.119 & 0.02 \\
\hline WT/WD B1 6th generation & $0.13(-0.10-0.34)$ & 36.53 & 27.95 & 0.194 & 0.02 \\
\hline WT/WA B1 6th generation & $0.04(-0.18-0.26)$ & 3.45 & 7.38 & 0.641 & $<0.01$ \\
\hline WT/WD B8 4th generation & $0.04(-0.18-0.26)$ & 37.28 & 43.79 & 0.396 & $<0.01$ \\
\hline WT/WA B8 4th generation & $0.01(-0.21-0.23)$ & 21.89 & 29.97 & 0.467 & $<0.01$ \\
\hline WT/WD B8 5th generation & $-0.08(-0.30-0.14)$ & -16.28 & 49.75 & 0.744 & $<0.01$ \\
\hline WT/WA B8 5th generation & $-0.01(-0.23-0.21)$ & 8.04 & 22.97 & 0.727 & $<0.01$ \\
\hline WT/WD B8 6th generation & $0.05(-0.17-0.27)$ & 13.78 & 27.75 & 0.620 & $<0.01$ \\
\hline WT/WA B8 6th generation & $0.19(-0.03-0.39)$ & 10.96 & 8.25 & 0.187 & 0.02 \\
\hline WT/WD B10 4th generation & $0.29(0.07-0.48)$ & 101.31 & 29.78 & 0.001 & 0.12 \\
\hline WT/WA B10 4th generation & $0.21(-0.01-0.42)$ & 82.83 & 35.96 & 0.023 & 0.06 \\
\hline WT/WD B10 5th generation & $0.23(0.01-0.43)$ & 86.35 & 37.01 & 0.022 & 0.06 \\
\hline WT/WA B10 5th generation & $0.18(-0.05-0.39)$ & 69.49 & 33.29 & 0.039 & 0.05 \\
\hline WT/WD B10 6th generation & $0.12(-0.10-0.34)$ & 43.38 & 34.63 & 0.213 & 0.02 \\
\hline WT/WA B10 6th generation & $0.04(-0.19-0.26)$ & 6.05 & 23.15 & 0.794 & $<0.01$ \\
\hline$L_{950} \%$ & $0.08(-0.12-0.29)$ & 0.01 & 0.01 & 0.429 & $<0.01$ \\
\hline Pi10 & $0.06(-0.17-0.28)$ & 0.49 & 0.40 & 0.227 & 0.01 \\
\hline
\end{tabular}

$\mathrm{FEV}_{1}$ : forced expiratory volume in $1 \mathrm{~s}$; FVC: forced vital capacity; $\mathrm{MEF}_{50 \%}$ : midexpiratory flow at $50 \%$ FVC; $\mathrm{MEF}_{25-74 \%}$ : midexpiratory flow at 25-75\% FVC; RV: residual volume; FRC: functional residual capacity; TLC: total lung capacity; $\mathrm{CV}$ : closing volume; $\mathrm{dN}_{2}$ : phase III nitrogen slope; $\mathrm{E} / \mathrm{I}_{\mathrm{MLD}}$ : expiratory/inspiratory ratio of mean lung density; WT: wall thickness; WD: wall diameter; B1: right apical bronchus; WA: wall area; B8: anterior basal bronchus; B10: posterior basal bronchus; I $_{950}$ : proportion of low attenuation below the threshold of $-950 \mathrm{HU}$ in the whole inspiratory volume; Pi10: square root of WA for a standardised airway with an internal perimeter of $10 \mathrm{~mm}$.

PFT and/or spirometrically controlled chest CT, and were thus excluded. Subjects were also excluded when PFT abnormalities were suggestive of undiagnosed lung disease according to American Thoracic Society/ European Respiratory Society criteria. The final study group consisted of 89 individuals with a mean $\pm \mathrm{SD}$ age of $71.7 \pm 7.9$ years including 52 women and 28 former light smokers (mean 0.4 pack-years). Automated airway tree skeletonisation and proximal airway segmentation were achievable for 81 (91\%) inspiratory CT scans. Air trapping calculation was obtained using both expiratory and inspiratory image sets.

Results of a univariate analysis of the relationship between airway measures and age, together with other clinical characteristics, are presented in table 1. Of the different airway measurements, only the LA values obtained at the fourth generation of B10 were related to age $(p=0.03)$, together with the WD/WT and WT/WA ratios in $\mathrm{B} 10$ at the fourth and fifth generations $(\mathrm{p}<0.05)$. No relationship was found for the other parameters of airway dimensions or Pi10 values. CT air trapping was correlated to age $(\mathrm{p}<0.0001$, correlation coefficient $0.48, \mathrm{R}^{2}=0.2$ ). The MLD of the whole inspiratory lung volume was $831.59 \pm 19.25 \mathrm{HU}$ and the proportion of voxels below the $-950-\mathrm{HU}$ threshold was $0.98 \pm 0.87 \%$; neither was related to age $(\mathrm{p}=0.21$ and $\mathrm{p}=0.42$, respectively). 
In this study, which explored the impact of ageing on airway remodelling, we found ageing mainly affected the distal airways, with demonstration of a close relationship between CT-quantified air trapping and age in healthy volunteers $>60$ years of age. Our findings suggest that structural changes are heterogeneous and that the ageing process, which involves the whole airway tree, is more pronounced at the level of the distal airways. The relationship between SBNT-derived parameters and age corroborates the prominent role of distal remodelling [10].

We did not identify a linear progression of wall thickening related to age between the fourth and the sixth generation as that suggested to exist in COPD [11]. Moreover, Pi10 was not related to age in our population. This suggests that physiological ageing and COPD affect the airway tree differently.

This study had several limitations. Only three segments between the fourth and the sixth generation in the right lung were analysed to assess a potential regional heterogeneity of the ageing process at the proximal level. For our sampling, we chose to evaluate bronchi away from paracardiac segments to avoid bronchial wall blurring due to cardiac motion artefacts. We did not perform bronchial measurements beyond the sixth generation because of their reported unreliability [12]. The second limitation is that expiratory CT was limited to a four-slice CT acquisition and did not consist of a volumetric whole-lung expiratory CT acquisition, in order to minimise the radiation exposure of our healthy volunteers. However, the four-slice sampling was sufficient to measure air trapping, which correlated well with functional indexes of small airway obstruction for each of the four levels [4].

Lastly, we were not able to elucidate the mechanism of distal airway obstruction reflected by air trapping, due to the limits of CT resolution. We might suspect it is due to bronchial thickening, as this phenomenon was found at a more proximal level, but we cannot affirm it is the main or unique cause of distal obstruction. In conclusion, the present study involving an asymptomatic healthy population aged between 60 and 90 years suggests that airway remodelling with age is a heterogeneous process having a particular tropism towards distal airways. Knowledge of this process can help distinguishing early changes in COPD or other diseases affecting the distal airways from normal ageing.

0 @ERSpublications

Airway remodelling with age is a heterogeneous process having a particular tropism towards distal airways http://ow.ly/FeZnN

Sébastien Bommart ${ }^{1,2,3}$, Grégory Marin ${ }^{4}$, Arnaud Bourdin ${ }^{2,3,5}$, Marie Pierre Revel ${ }^{6}$, François Klein ${ }^{1}$, Maurice Hayot ${ }^{2,3,7}$, Isabelle Vachier, Pascal Chanez, Marie Christine Picot ${ }^{4}$, Jacques Mercier ${ }^{2,3,7}$, Nicolas Molinari ${ }^{4}$ and Hélène Vernhet-Kovacsik ${ }^{1}$

${ }^{1}$ Radiology Dept, Centre Hospitalier Universitaire (CHU) Montpellier, Montpellier, France. ${ }^{2}$ INSERM U 1046, Université Montpellier 1, Montpellier, France. ${ }^{3}$ Université Montpellier 2, Montpellier, France. ${ }^{4}$ Biostatistics Dept, CHU Montpellier, Montpellier, France. ${ }^{5}$ Respiratory Disease Dept, CHU Montpellier, Montpellier, France. ${ }^{6}$ Radiology Dept, Cochin Hospital, Université Paris Descartes, Paris, France. ${ }^{7}$ Clinical Physiology Dept, CHU Montpellier, Montpellier, France. ${ }^{8}$ Université Aix-Marseille, Marseille, France.

Correspondence: Sébastien Bommart, CHU Montpellier, 371 avenue Doyen Gaston Giraud, 34295, Montpellier cedex 05, France. E-mail: s-bommart@chu-montpellier.fr

Received: Oct 032014 | Accepted after revision: Nov 252014 | First published online: Dec 232014

Support statement: The study was funded by a grant from the French Ministry of Health (Programme Hospitalier de Recherche Clinique A00810-55).

Conflict of interest: None declared.

Acknowledgements: We thank all the volunteers who participated in this research study; Melina Bouet, Claire Belloc and Erika Nogue for logistical assistance and data management; and Pamela Goodman-Stephens for her linguistic contribution (all CHU Montpellier, Montpellier, France).

\section{References}

1 Vestbo J, Hurd SS, Agusti AG, et al. Global strategy for the diagnosis, management, and prevention of chronic obstructive pulmonary disease: GOLD executive summary. Am J Respir Crit Care Med 2013; 187: 347-365.

2 MacNee W, Rabinovich RA, Choudhury G. Ageing and the border between health and disease. Eur Respir J 2014; 44: 1332-1352.

3 Lepeule J, Bind MA, Baccarelli AA et al. Epigenetic influences on associations between air polluants and lung function in elderly men: the normative aging study. Environ Health Perspect 2014; 122: 566-572.

4 Janssens JP, Pache JC, Nicod LP. Physiological changes in respiratory function associated with ageing. Eur Respir J 1999; 13: 197-205.

5 Grydeland TB, Dirksen A, Coxson HO, et al. Quantitative computed tomography: emphysema and airway wall thickness by sex, age and smoking. Eur Respir J 2009; 34: 858-865.

6 Bommart S, Marin G, Bourdin A et al. Relationship between CT air trapping criteria and lung function in small airway impairment quantification. BMC Pulm Med 2014; 28: 14-29. 
7 Quanjer PH, Stanojevic S, Cole TJ, et al. Multi-ethnic reference values for spirometry for the 3-95-yr age range: the global lung function 2012 equations. Eur Respir J 2012; 40: 1324-1343.

8 Bourdin A, Paganin F, Prefaut C, et al. Nitrogen washout slope in poorly controlled asthma. Allergy 2006; 61: 85-89.

9 Robinson PD, Latzin P, Verbanck S, et al. Consensus statement for inert gas washout measurement using multiple- and single- breath tests. Eur Respir J 2013; 41: 507-522.

10 Burgel PR, Bourdin A, Chanez P, et al. Update on the roles of distal airways in COPD. Eur Respir Rev 2011; $20: 7-22$.

11 Hasegawa M, Nasuhara Y, Onodera Y, et al. Airflow limitation and airway dimensions in chronic obstructive pulmonary disease. Am J Respir Crit Care Med 2006; 173: 1309-1315.

12 Takahashi M, Okada H, Oguni N, et al. How accurate is CT morphometry of airway? Phantom and clinical validation study. Eur J Radiol 2011; 80: e524-e530.

\title{
Monitoring toxicity in individuals receiving treatment for latent tuberculosis infection: a systematic review versus expert opinion
}

\author{
To the Editor:
}

Latent tuberculosis infection (LTBI) is defined as a state of persistent immune response to stimulation by Mycobacterium tuberculosis antigens without evidence of clinically manifested active TB [1]. The World Health Organization (WHO) estimates that one-third of the global population is infected by Mycobacterium tuberculosis [2]. They cannot transmit tuberculosis (TB), but they can develop the disease and become infectious with a life-time risk of approximately 5-15\% [3]. A significant proportion of new TB patients, especially in low burden countries, are presumed to occur from the pool of individuals with LTBI.

Treatment of LTBI by isoniazid and/or rifamycin can reduce the risk of future disease by 60-90\% [4]. Systematic testing and treatment of LTBI is recommended in several at-risk populations with a favourable trade-off between benefits and harms of treatment. As the majority of individuals undergoing LTBI treatment would never develop $\mathrm{TB}$, regardless of therapy, it is mandatory to minimise risks during treatment. Specifically, it is imperative to design a mechanism to regularly monitor and manage adverse events.

Drug-specific adverse reactions can occur with isoniazid (asymptomatic elevation of serum liver enzyme concentrations, peripheral neuropathy and hepatotoxicity); rifampicin and rifapentine (cutaneous reactions, hypersensitivity reactions, gastrointestinal intolerance and hepatotoxicity). A meta-analysis involving 38257 subjects treated with isoniazid, estimated the risk of clinical hepatitis at six per 1000 treated individuals (range among the six studies: 0.0-2.9\%) [5]. Later, the risk of isoniazid-related symptomatic hepatitis was estimated as $1-3$, that of mortality $0-0.3$ and that of hospitalisation $0.1-0.2$ per 1000 treated persons [6]. More recently, among 24221 participants in a large trial that was conducted in South Africa, 132 possible adverse events (0.54\%) were reported among 130 individuals. Clinical hepatotoxicity was associated with the consumption of alcohol [7]. Evidence shows that the rate of adverse events, in particular death, decreased with time, probably due to better clinical care [8].

We performed a systematic review on the best clinical approach to monitor toxicity in individuals treated for LTBI to inforzm the WHO process for the development of the LTBI management guidelines [9]. The outcome measurements were: mortality and morbidity related to toxicity and tolerability of the preventive therapy. All the outcomes would have been evaluated under different proposed monitoring conditions (i.e. "wait and see", clinical and/or biochemical monitoring).

All pertinent studies, limited to English, French and Spanish languages, regardless of geographical setting, were considered. The search had no time limitations and included the following keywords combined in specific strings: TB, LTBI, toxicity, adverse events, safety, tolerability, anti-TB drugs, and preventive therapy. The search was conducted in the electronic databases PUBMED and EMBASE, and, then, expanded to the reference lists of relevant studies and review articles. All the retrieved records were electronically collected and selected on the basis of pre-defined inclusion criteria i.e. study design (cross-sectional, case-control, and retrospective/prospective cohort studies or clinical trials), type of participants (individuals with suspected LTBI, tuberculin skin test and/or interferon- $\gamma$ positive release 